\title{
Por Que os Estados Querem a Bomba? As Condições Para a Proliferação Nuclear
}

\author{
Why do States Want the Bomb? \\ The Determinants for Nuclear Proliferation
}

RAQUEL GONTIJO

\section{INTRODUÇÃO}

A explosão das bombas atômicas em 1945 representou um salto qualitativo na capacidade humana de destruição. ${ }^{1}$ Por consequência, a difusão dessa tecnologia tornou-se um dos principais temas da agenda internacional, e ainda ocupa, mais de meio século depois, um lugar de destaque nos debates tanto entre analistas quanto entre atores políticos. ${ }^{2}$ Desde 1945 , oito novos países adquiriram capacidade nuclear, e vários outros exploraram essa opção de forma relativamente séria. ${ }^{3}$

No entanto, ao nos perguntarmos por que os Estados desejam obter a bomba, nos deparamos com respostas diversas: porque eles têm medo, porque ambicionam maior prestígio, ou simplesmente porque eles têm a capacidade. De fato, para responder a essa pergunta, pesquisadores têm apresentado diversas hipóteses, com base em concepções teóricas, em inferências estatísticas e em levantamentos históricos.

Esse tema, que motivou um amplo debate internacional, é ainda pouco explorado na academia brasileira, conquanto o Brasil seja frequentemente identificado como um dos países que já buscaram desenvolver armamentos nucleares (Cirincione 2007; Jo, Gartzke 2006, 2007; Montgomery, Sagan 1997, 2011). De fato, as pesquisas desenvolvidas no Brasil sobre política nuclear têm como foco, em geral, o programa nuclear brasileiro, a questão da proliferação nas interações regionais e o posicionamento do Brasil frente aos regimes de não proliferação nuclear (Jesus 2012; Medeiros 2005; Oliveira 1998; Patti 2013; Silva 2010; Vargas 1997). ${ }^{4}$

Este artigo tem por objetivo sistematizar algumas das principais interpretações sobre as motivações que levam os Estados a buscarem arma-

Raquel Gontijo - Doutoranda em Relações Internacionais pelo Programa de Pós-Graduação San Tiago Dantas (UNESP, UNICAMP e PUC-SP).E-mail: raquelbgontijo@gmail.com. 
mentos nucleares. Há várias formas de agrupar essas hipóteses. Scott D. Sagan (1997), por exemplo, em seu muito citado artigo Why do states build nuclear weapons?, apresenta três modelos $^{5}$ para a compreensão das causas da proliferação: o modelo da segurança, o modelo da política doméstica, e o modelo das normas. Já em trabalho posterior, Sagan (2011) agrupa as causas da proliferação em dois grandes grupos: as causas associadas à oferta e as causas associadas à demanda. Thomas Graham Jr. e Keith A. Hansen (2009, 7-2 1) apresentam apenas duas motivações para um Estado desejar a bomba, segurança nacional e prestígio. Sonali Singh e Christopher R. Way (2004) derivam suas hipóteses de três grandes abordagens sobre as causas da proliferação: os determinantes tecnológicos, os determinantes externos e os determinantes internos (domésticos). Joseph Cirincione (2007, 47-83) propõe cinco categorias de motivadores da proliferação: segurança, prestígio, política doméstica, tecnologia e economia. ${ }^{6}$ Tanya Ogilvie-White (2008) agrupa os as causas da proliferação em modelos das teorias realistas e neorrealistas, de determinantes domésticos e teorias organizacionais, de aspectos cognitivos e psicológicos e da sociologia histórica.

Outras divisões podem ser encontradas na literatura, mas os exemplos acima já deixam clara a diversidade de abordagens e a recorrência de alguns elementos. Optamos, aqui, por dividir os modelos sobre as causas ${ }^{7} \mathrm{da}$ proliferação nuclear em quatro grandes grupos, na tentativa de englobar, de forma simples, as várias teorias e hipóteses que fazem parte do campo: modelos que partem da demanda por segurança, modelos que partem do compartilhamento de normas e ideias, modelos que partem da política doméstica ${ }^{8}$ e modelos que partem da oferta de tecnologia. ${ }^{9}$

Deve ser observado que, inevitavelmente, é inviável incorporar todas as nuances que surgem nesses debates. Nosso propósito não é oferecer uma revisão exaustiva sobre as causas da proliferação, mas, antes, apresentar as principais contribuições que têm sido feitas aos estudos desse campo, acompanhadas de algumas das evidências identificadas por pesquisadores para corroborar cada modelo. Quando pertinente, apontaremos os pontos de conflito e desacordo entre modelos e entre autores. No entanto, é fundamental que fique clara a complementariedade dos diversos modelos: não se trata de uma disputa para definir um único modelo que consiga explicar todo o histórico de proliferação nuclear; trata-se da busca coletiva por uma melhor compreensão de um fenômeno que é, necessariamente, complexo e multicausal.

Na próxima seção, abordaremos o modelo mais tradicional sobre as causas da proliferação, partindo da demanda dos Estados por maior segurança e, por conseguinte, por melhores armamentos. Em seguida, apresentaremos o modelo baseado no compartilhamento de normas e ideias. A 
quarta seção discute os condicionantes que emergem da política doméstica e da disputa entre grupos de interesse. $\mathrm{O}$ último modelo, que explora as condições econômicas, científicas e tecnológicas que propiciam a proliferação será discutido na quinta seção. Finalmente, concluímos o artigo com algumas reflexões sobre o debate acerca das condições que permitem ou estimulam a proliferação.

\section{A DEMANDA POR SEGURANÇA}

O modelo mais tradicional sobre o que leva à proliferação está associado à teoria realista de Relações Internacionais, e aponta a insegurança inerente ao sistema como o principal motivador para que Estados desejem e busquem armamentos nucleares (Cirincione 2007; Graham Jr., Hansen 2009; Jo, Gartzke 2007; May 1994; Ogilvie-White 1996; Sagan 1996/97, 2011; Thayer 1995).

Esse imperativo de segurança sugere que Estados que enfrentem ameaças significativas em seu contex to de segurança estão mais propensos a buscarem um arsenal nuclear. Tais ameaças podem decorrer tanto de inimigos que já detêm capacidade nuclear, como é o caso do Paquistão após a explosão da bomba indiana, ${ }^{10}$ quanto de inimigos sem capacidade nuclear, mas com forças convencionais expressivas, como ocorre no contexto de segurança de Israel, cujos inimigos, além de numerosos, estão muito próximos de seu território. Assim, um Estado que se sinta ameaçado pode ambicionar seu próprio arsenal nuclear com o intuito de dissuadir potenciais agressores.

Michael M. May descreve da seguinte forma a relação entre segurança e proliferação:

Nações que tomaram a decisão de ter armamentos nucleares ou perceberam uma ameaça à sua sobrevivência que nenhum outro Estado poderia dissuadir com credibilidade, ou enfrentaram uma situação em que, com a capacidade nuclear, poderiam aumentar significativamente sua voz em assuntos relevantes para sua sobrevivência ${ }^{11}$ (May 1994, 534, tradução nossa).

Os argumentos associados ao modelo do imperativo de segurança, desenvolvidos por diferentes analistas, não são homogêneos, mas todos os proponentes desse modelo identificam a busca dos Estados por melhores condições de se defenderem contra ameaças à sua sobrevivência como um fator central para a compreensão da proliferação. Nesse sentido, Bradley A. Thayer $(1995,486)$ afirma que o imperativo de segurança é o único elemento capaz de explicar todos os casos de proliferação, sendo condição 
necessária e suficiente para que a proliferação ocorra, e que os demais modelos podem apenas contribuir para a compreensão de casos históricos específicos. Similarmente, Monteiro e Debs (2014) argumentam que a insegurança é condição necessária para a proliferação, uma vez que todos os países que adquiriram armamentos nucleares enfrentavam ameaças à sua sobrevivência. ${ }^{12}$ No entanto, há outros autores que assumem uma postura mais parcimoniosa, identificando o imperativo de segurança como apenas uma das condições que propiciam a proliferação, e não a única (Betts 1977; Cirincione 2007; Epstein 1977; Graham Jr., Hansen 2009; Sagan 1996/97).

Esse modelo encontra amplo respaldo nas análises sobre o tema: os estudos de Sonali Singh e Christopher R. Way (2004) e de Dong-Joon Jo e Erik Gartzke (2007) demonstram que considerações de segurança são fundamentais para a compreensão da proliferação, uma vez que Estados engajados em longas rivalidades, ou envolvidos em conflitos militares frequentes, têm maior probabilidade de se tornarem proliferadores. Paralelamente, garantias de defesa oferecidas por aliados com capacidade nuclear (comumente chamadas de "guarda-chuva nuclear") reduzem o risco de proliferação. Similarmente, o imperativo de segurança é identificado como o motivador de muitos programas nucleares, incluindo os programas dos Estados Unidos, Rússia, China, França, Reino Unido, Paquistão, Israel, África do Sul, Coreia do Sul, Iraque e Líbia (Betts 1977; Cirincione 2007; Graham Jr., Hansen 2009; May 1994; Sagan 1996/97).

$\mathrm{O}$ caso indiano oferece um exemplo curioso, devido à dificuldade de atribuir seu programa nuclear a uma única motivação. Vários autores entendem a busca indiana pela bomba como uma resposta à sua insegurança frente a potenciais inimigos externos, em conformidade com o modelo do imperativo de segurança. A derrota sofrida na disputa fronteiriça com a China, em 1962, e o subsequente teste da bomba chinesa, em 1964, podem ser interpretados como importantes estímulos para o programa nuclear indiano, que culminaria no teste de uma explosão nuclear "pacífica” em 1974 (Frankel 1993). Além disso, alterações na dinâmica política regional no pós-Guerra Fria teriam levado a um aumento da percepção de ameaça associada tanto à China quanto ao Paquistão (Ganguly 1999; Weixing 2000). Assim, a relativa fraqueza da Índia frente a inimigos externos, unida à baixa credibilidade das garantias de segurança oferecidas por seus aliados com capacidade nuclear, podem ter sido as motivações centrais para que a Índia ingressasse no clube nuclear (Monteiro, Debs 2014). No entanto, como se verá adiante, vários analistas compreendem a bomba indiana a partir de outras matrizes explicativas.

Com efeito, não obstante o forte amparo que o modelo do imperativo de segurança encontra na literatura, muitos autores consideram que ele não 
é suficiente para contemplar todo o histórico da proliferação (e.g. Lavoy 1993; Solingen 1994). Sagan (1996/97) oferece dois questionamentos particularmente pertinentes ao imperativo de segurança: 1) os analistas parecem se apoiar muito no discurso oficial de justificação dos programas nucleares, o que gera distorções, na medida em que os governos têm incentivos para alegar uma ameaça à sua sobrevivência como motivação principal; e 2) é possível perceber uma tendência problemática de construção de análises post hoc, em que os analistas selecionam um Estado proliferador e, em seguida, buscam identificar a ameaça que motivou sua política nuclear.

O desenvolvimento de outros modelos permitiu, então, uma melhor compreensão das forças motrizes da proliferação, contribuindo para preencher as lacunas que o modelo da demanda por segurança não era capaz de contemplar. Um desses modelos alternativos busca no contex to normativo e ideacional elementos que nos permitam compreender a proliferação, como será visto a seguir.

\section{AS IDEIAS COMPARTILHADAS}

A segunda forma de compreender a proliferação nuclear se pauta por uma interpretação normativa das Relações Internacionais. Essa interpretação, fundamentada nas contribuições sociológicas e construtivistas para o campo, enfatiza a importância das normas e ideias compartilhadas para a condução da política internacional e a definição dos cursos de ação adotados pelos atores (estatais ou não). Dentre esses cursos de ação influenciados por fatores ideacionais, inclui-se a decisão dos Estados de desenvolverem armamentos nucleares, decisão que estaria associada a percepções (intersubjetivas) de legitimidade e de poder. Assim, na medida em que os Estados considerarem legítimo o desenvolvimento nuclear, e o associarem às características determinantes de uma grande potência, a escolha de buscar a bomba será mais provável (Chafetz, Abramson, Grillot 1996; Cirincione 2007; Egeland 2016; Epstein 1977; Graham Jr., Hansen 2008; Hymans 2006b; Price, Tannenwald 1996; Sagan 1996/97; Suchman, Eyre 1992; Tannenwald 2007).

Sob a perspectiva normativa e ideacional, portanto, a história da proliferação nuclear está intimamente associada a noções de prestígio internacional. Estados ambicionam a bomba, portanto, porque percebem a posse de um arsenal nuclear como uma forma de adquirir prestígio frente aos demais atores e de aumentar seu poder político nas interações internacionais (Betts 1977; Cirincione 2007; Egeland 2016; Epstein 1977; Sagan 1996/97). Assim, no contexto da década de 1960, por exemplo, havia uma percepção difundida de que a posse de um arsenal nuclear permitiria a um 
Estado fazer parte do seleto grupo de potências internacionais. Isso teria sido fundamental para que países como a França, cuja identidade era historicamente associada à sua posição de potência, e como a Índia, que adotava, já então, uma postura de país emergente, desenvolvessem sua capacidade nuclear. Distintamente, com a consolidação do Regime Internacional de Não Proliferação, principalmente a partir da década de 1990, o contexto normativo foi de tal forma modificado que a rejeição à bomba passou a implicar um novo tipo de prestígio internacional. Assim, a adesão às normas de não proliferação e o repúdio ao desenvolvimento ilegal da bomba nuclear passaram a ser associados a um papel de "bom cidadão", que vários Estados optam por adotar em suas relações internacionais (Chafetz, Abramson, Grillot 1996). Paralelamente, a ambição por um arsenal nuclear passou a ser associada a Estados pária, como o Iraque, no começo da década de 1990, e a Coreia do Norte, que persiste em uma situação de profundo isolamento internacional (Klare 2011; O'Reilly 2013).

Nesse contexto, se armamentos nucleares são imbuídos de valor tão elevado, é preciso também uma explicação normativa para sua lenta difusão. De fato, Nina Tannenwald (2007) argumenta que, ao longo da Guerra Fria, formou-se um tabu relacionado ao uso de armamentos nucleares, o que contribuiu para a ampliação do repúdio à própria posse desses armamentos (ao menos, pelos países não legitimados como potências nucleares no Tratado de Não Proliferação, de 1968). Segundo Tannenwald (2007), o tabu seria um tipo específico de norma proibitiva, associada a uma noção de perigo. De fato, o tabu que se desenvolveu imporia a proibição do primeiro emprego da bomba, uma vez que seu uso, mesmo que apenas uma vez, significaria cruzar uma linha invisível, uma barreira concebida para proteger a humanidade (Price, Tannenwald 1996; Tannenwald 2007). Thomas Schelling (1980) expressa um argumento semelhante, sem empregar a noção de tabu: o que difere os armamentos nucleares dos explosivos convencionais não é uma característica intrínseca, mas uma tradição de que alguma diferença substancial existe, o que levou à construção de uma “ $[\ldots]$ expectativa conjuntamente reconhecida de que [armamentos nucleares] não podem ser usados [...]"13 (Schelling 1980, 260, tradução nossa).

Os defensores desses modelos apontam vários exemplos de países cuja postura nuclear foi influenciada de forma determinante por essas considerações normativas: a França e o Reino Unido, por exemplo, seriam países que optaram por desenvolver a bomba em busca de prestígio (Cirincione 2007; Ogilvie-White 1996; Sagan 1996/97). Paralelamente, a África do Sul, a Ucrânia, a Líbia e a Austrália seriam países que rejeitaram a bomba, motivados pelas normas compartilhadas do sistema (Cirincione 2007; Hymans 2006b; Ogilvie-White 1996; Sagan 1996/97). 
A Índia também é apontada como um exemplo de Estado cuja postura nuclear foi fortemente influenciada pelo desejo de prestígio. No entanto, curiosamente, enquanto o argumento mais comum parece indicar que tal desejo resultou no desenvolvimento do programa nuclear indiano (Cirincione 2007; Epstein 1977; Sagan 1996/97), Tanya Ogilvie-White (1996), escrevendo antes do teste de 1998, aponta as considerações normativas como um motivador para que a Índia abandonasse seu programa nuclear.

O modelo ideacional e normativo elucida aspectos fundamentais do processo de proliferação que não são comportados pelos modelos tradicionais baseados apenas em aspectos materiais. Entretanto, esse modelo enfrenta sérios desafios em sua operacionalização. Identificar, de forma confiável, quais normas efetivamente estão atuando em um sistema pode estar ainda além da capacidade dos analistas, uma vez que a pluralidade de visões e de posições individuais gera um ambiente de extrema complexidade ideacional. Além disso, Sagan (2004) questiona a possibilidade de se falar em um tabu nuclear, percebendo, antes, a existência de uma "tradição de não uso", informada não pela estrutura normativa ou por considerações morais, mas pelo cálculo frio de custos e benefícios. Os Estados seriam, então, orientados pela percepção de que o emprego da bomba seria maléfico para o próprio país que a empregasse, uma vez que estabeleceria um precedente de uso, que poderia ser acionado por outros países em contextos posteriores. Finalmente, Peter Lavoy (1993) percebe a busca por prestígio como uma explicação residual, adotada por analistas para aqueles casos em que as motivações materiais não estão claras.

Parece certo que ainda são necessárias pesquisas mais robustas sobre como as normas vigentes no setor nuclear são construídas e o mecanismo através do qual elas impactam sobre a proliferação. Além disso, permanecem divergências substanciais entre os pesquisadores sobre em que medida é possível atribuir os programas nucleares de países como a Índia, a França e o Reino Unido a considerações normativas.

Algumas dessas considerações normativas podem ser melhor aplicadas quando olhamos não para os Estados como entidades únicas, mas para sua dinâmica interna e as interações entre indivíduos e grupos. A próxima seção apresenta, então, o modelo que trata do impacto da política doméstica sobre as escolhas nucleares.

\section{A POLÍTICA DOMÉSTICA}

O terceiro modelo sobre as causas da proliferação olha para dentro dos Estados em busca dos elementos que levam à decisão de desenvolver armamentos nucleares. Este modelo se baseia, sobretudo, na análise fundacional 
desenvolvida por Graham Allison (1969) e por Morton Halperin (1972). Segundo Allison e Zelikow (1971), a política doméstica é necessária para a compreensão da política internacional, uma vez que as decisões dos governos não são fruto de uma única liderança com posições coerentes e racionais, mas, antes, são resultado da interação entre atores em diferentes níveis de atuação. Dentro dos Estados, interagem organizações e burocracias, que têm interesses, objetivos e padrões de procedimentos específicos. ${ }^{14}$ As organizações influenciam a forma como um determinado tema é percebido e interpretado pelo governo, uma vez que elas captam e processam as informações. Além disso, elas determinam, em alguma medida, as opções políticas que estão disponíveis para um país, na medida em que essas opções são constrangidas pelos recursos materiais e humanos que podem ser mobilizados por um governo através dessas organizações.

Além dos procedimentos operacionais das organizações, as políticas adotadas por um país são influenciadas pela interação política entre membros do governo, cujos diferentes interesses são disputados através de canais institucionalizados de barganha. ${ }^{15}$ Atores específicos, constrangidos pelos cargos que ocupam, determinam, então, os rumos das decisões governamentais, através de suas negociações, coalizões, acordos e desacordos (Allison 1969; Allison, Zelikow 1971). Essa perspectiva se ampara no reconhecimento de que a política é feita por indivíduos:

Homens compartilham poder. Homens têm visões diferentes sobre o que deve ser feito. Essa diferença importa. Esse ambiente requer que as políticas públicas sejam resolvidas através da interação política. O que uma nação faz é, às vezes, resultado do triunfo de um grupo sobre os demais ${ }^{16}$ (Allison 1969, 707, tradução nossa).

Ao ser aplicado à análise das condições que levam à proliferação, este tipo de abordagem sugere que Estados terão maior inclinação a se tornarem proliferadores quando grupos internos favoráveis à aquisição da bomba formarem uma coalizão suficientemente forte para direcionar os rumos da decisão governamental. Três grupos são centrais nesse processo: os cientistas associados à área nuclear, os militares e os políticos. Enquanto tanto cientistas quanto militares têm a ganhar em termos de alocação de recursos e elevação do prestígio institucional, atores do meio político podem perceber ganhos eleitorais e aumento de popularidade associados a uma postura nuclear mais forte (Cirincione 2007; Sagan 1996/97; Solingen 1994).

Neste contexto, a existência de criadores de mitos nucleares (nuclear myth makers) pode ser fundamental para que as lideranças políticas adotem uma postura favorável ao desenvolvimento da bomba. Esses criadores de mitos são indivíduos posicionados de forma privilegiada em termos polí- 
ticos, que conseguem difundir um discurso favorável ao desenvolvimento nuclear. Esse discurso exagera os benefícios associados à bomba, realçando os ganhos de segurança e prestígio internacional, e atenua os custos associados à sua produção (Lavoy 1993).

Similarmente, Stephen Flank (1993) propõe uma análise sociológica do avanço tecnológico, destrinchando a complexidade dos elementos sociais que decorrem de um programa de desenvolvimento e produção de uma tecnologia. Assim, a tecnologia passa a ser vista como um ator no cenário político, ator este que busca adquirir aliados que permitam a continuidade dos projetos de desenvolvimento tecnológico.

Dentre os casos apontados por proponentes desse modelo como exemplos de países cujas políticas nucleares se devem a elementos de política doméstica estão a África do Sul, o Paquistão, o Japão, a Argentina e o Brasil (Cirincione 2007; Flank 1993; Lavoy 1993; Sagan 1996/97). Aqui, novamente, a Índia aparece como um exemplo que pode oferecer evidências condizentes com este modelo de análise sobre a proliferação. De fato, o papel desempenhado por Homi Bhaba, diretor do programa nuclear indiano, é amplamente reconhecido como fundamental para que a Índia produzisse a bomba (Cirincione 2007; Flank 1993; Ganguly 1999; Sagan 1996/97), e Lavoy (1993) o cita como um exemplo icônico de um criador de mito nuclear.

Mais notavelmente nos dias atuais, o programa nuclear da Coreia do Norte parece ser fortemente influenciado por dinâmicas políticas domésticas (Nakatani 2012; Woo 2015) ${ }^{17}$ A necessidade do regime dos Kim de se legitimar e perpetuar frente às diferentes pressões domésticas sugere que a postura nuclear norte-coreana visa a transmitir uma imagem de força por parte do governo. Essa interpretação recebeu força com a realização, em maio de 2016, da primeira convenção do Partido dos Trabalhadores da Coreia em mais de 35 anos, a qual teve como função fortalecer a posição de Kim Jong Un na liderança do país (Lee 2016).

Apesar da inegável relevância da política doméstica para a compreensão de um fenômeno como a proliferação, este modelo sofre de uma fragilidade similar à apontada para o modelo normativo: sua dificuldade de operacionalização. De fato, pode ser fácil identificar, a posteriori, a presença de criadores de mitos nucleares, mas esse modelo analítico dificilmente pode oferecer um melhor discernimento sobre tendências futuras e, por conseguinte, terá pouco a contribuir em termos de formulação de políticas de não proliferação. Há também uma carência de teorização sobre as condições que permitem a formação de coalizões favoráveis à bomba dentro de um país e, mais além, as condições que permitem seu sucesso no embate político doméstico (Sagan 1996/97). Além disso, os analistas ainda encontram dificuldade em identificar quais indivíduos realmente importam para 
o jogo político, em traçar suas opiniões ao longo do tempo e, principalmente, em diferenciar os discursos formulados como justificativas para determinada decisão política daqueles que realmente expressam as motivações por trás dessa decisão (Chafetz, Abramson, Grillot 1996).

Até aqui, discutimos os elementos que têm impacto sobre a demanda dos Estados pela bomba e sua disposição em promover um programa de desenvolvimento nuclear, incluindo aspectos materiais e ideacionais, nos ambientes internacional e doméstico. A próxima seção volta-se para o outro lado desse processo, identificando a importância da oferta para a compreensão da proliferação.

\section{A OFERTA}

Um Estado pode ambicionar intensamente a posse de um arsenal nuclear, mas, se ele não tiver as condições para desenvolver ou adquirir esse arsenal, tal ambição não resultará em proliferação. Assim, o determinante tecnológico é fundamental para a compreensão da difusão da capacidade nuclear. Esse determinante pode ser dividido em dois elementos distintos. Primeiro, a capacidade que um Estado tem de conduzir endogenamente um programa nuclear, ou seja, seu nível de desenvolvimento econômico, científico e industrial (Jo, Gartzke 2007; Sagan 201 1; Singh, Way 2004). Segundo, o acesso que um Estado tem a tecnologias e conhecimentos relevantes para o programa nuclear, através de acordos de cooperação nuclear civil e transferências internacionais (Braun, Chyba 2004; Cirincione 2007; Fuhrmann 2008, 2009a, 2009b; Kroenig 2009a, 2009b; May 1994; Sagan 2011).

Este modelo assume que, em condições de insegurança, será mais provável que os Estados iniciem a busca por armamentos nucleares caso os custos de seu desenvolvimento sejam amenizados pela cooperação internacional. ${ }^{18}$ Além disso, uma vez iniciada essa busca, os Estados terão melhores condições de serem bem-sucedidos em sua produção da bomba caso usufruam de parcerias internacionais. A partir disso, pode-se concluir que a escolha de se desenvolverem armamentos nucleares será influenciada, de forma decisiva, por limitações de recursos, sejam estes técnicos, humanos, financeiros ou políticos. Por conseguinte:

Porque os Estados têm a ganhar com a posse de armamentos nucleares, os fatores do lado da oferta que possibilitam o desenvolvimento nuclear estão entre os determinantes mais importantes da proliferação nuclear ${ }^{19}$ (Gartzke, Kroenig 2009, 152, tradução nossa).

Para Joseph Cirincione (2007), o determinante tecnológico atua mais como uma barreira à proliferação do que como um estímulo. Assim, paí- 
ses que em determinado momento desejaram obter a bomba, como a Argentina, o Brasil, o Irã e o Iraque, foram frustrados pela falta de acesso às tecnologias e conhecimentos necessários para o desenvolvimento da capacidade nuclear.

Contrariamente à visão de Cirincione (2007), no entanto, as análises mais intrigantes sobre o lado da oferta apontam para o papel que a cooperação internacional desempenha favorecendo a proliferação. Matthew Kroenig (2009b) demonstra a relação existente entre a transferência de tecnologias e conhecimentos sensíveis e a proliferação nuclear. $\mathrm{O}$ autor restringe assistência nuclear sensível a três pontos: assistência na concepção e no projeto de armamentos nucleares; transferência de material físsil que pode ser utilizado na fabricação de bombas nucleares; e, finalmente, auxílio no desenvolvimento e construção de instalações para produção de material físsil ${ }^{20}$ adequado para a fabricação de bombas nucleares. Partindo dessa qualificação, Kroenig (2009b) demonstra que a assistência sensível contribui significativamente para que um Estado embarque em um programa de armamentos nucleares porque: 1) a concepção do projeto de armamentos nucleares requer alto nível de sofisticação, impondo uma dificuldade de engenharia que pode ser mitigada, ou mesmo eliminada, através de parcerias com um Estado que já detenha um arsenal nuclear; 2) programas nucleares são fortemente marcados por tentativa e erro, o que leva a alto dispêndio de recursos materiais e de tempo, e a assistência sensível permite que vários erros sejam evitados; 3) a instalação da infraestrutura necessária para o desenvolvimento e a produção de armamentos nucleares tem custos elevados, que podem ser reduzidos através da assistência internacional; e 4) Estados que embarcam na busca da bomba nuclear são, em geral, alvos de forte pressão internacional, a qual pode ser mitigada pela parceria com Estados nucleares.

Por sua vez, Matthew Fuhrmann (2009a) desenvolve o argumento da oferta de forma distinta. Ele recusa a restrição proposta por Kroenig (2009b), de que apenas assistência em áreas sensíveis aumenta o risco de proliferação nuclear. Com efeito, Fuhrmann (2009a; 2009b) argumenta que qualquer tipo de cooperação na área nuclear, inclusive cooperação para fins unicamente civis, impacta sobre o risco de proliferação. Nas palavras do autor,

[...] como todas as commodities nucleares têm, por natureza, dupla utilidade (dual-use), Estados importadores podem usar a tecnologia e o conhecimento adquiridos para produzir armas nucleares ${ }^{21}$ (Fuhrmann 2009b, 5-6, tradução nossa).

Neste sentido, a assistência para fins civis não deve ser descartada como irrelevante para o problema da proliferação porque, assim como a assis- 
tência sensível, ela contribui para a redução dos custos de um programa nuclear, na medida em que permite a qualificação de pessoal, a aquisição de expertise e know-how e a instalação de infraestruturas. Assim, Fuhrmann (2009a) argumenta que os elevados custos de um programa de desenvolvimento de armamentos nucleares podem ser reduzidos até um patamar que os líderes políticos domésticos considerem aceitável, como efeito da cooperação civil. ${ }^{22}$ Sem dúvida, a análise de Fuhrmann (2009a) não leva à conclusão de que todos os países que receberem auxílio no campo nuclear buscarão adquirir a bomba. No entanto, alguns Estados poderão ser levados a buscar armamentos nucleares sob as novas condições, ainda que não o fizessem sem a redução dos custos decorrente da cooperação civil.

O modelo da oferta encontra respaldo nos estudos de Jo e Gartzke (2007) e de Singh e Way (2004), que trazem evidências de que o nível de desenvolvimento econômico e industrial de um país desempenha papel relevante na proliferação e, abaixo de um determinado patamar de desenvolvimento econômico, a proliferação é inviável e, portanto, não ocorre. Paralelamente, tanto Kroenig (2009b) quanto Fuhrmann (2009a) apresentam resultados estatísticos expressivos que corroboram a hipótese de que a cooperação internacional no setor nuclear para fins civis aumenta o risco da proliferação. De fato, a assistência internacional parece ter desempenhado papel importante nos programas nucleares de vários Estados, principalmente entre países em desenvolvimento, como a Coreia do Norte, Israel, Paquistão, Irã, Líbia e África do Sul (Braun, Chyba 2004; Fuhrmann 2009a; Kroenig 2009b). Para a Índia, a assistência internacional foi um componente de grande relevância, principalmente nos primórdios de seu programa nuclear. O país recebeu auxílio na forma de transferência de instalações e materiais, treinamento de pessoal e consultoria científica por parte dos Estados Unidos, do Canadá e do Reino Unido, o que pode ter sido crucial para que seu programa nuclear tivesse sucesso (Fuhrmann 2009a; Holdren 1983).

Apesar desse suporte empírico, o modelo da oferta de tecnologia também é alvo de críticas. Enquanto alguns analistas questionam uma versão radical (e, em estudos mais recentes, incomum) do modelo, segundo a qual todos os Estados com capacidade suficiente buscarão desenvolver a bomba, outros analistas desafiam o modelo de forma mais robusta. Esse é o caso de Alexander H. Montgomery (2013) e R. Scott Kemp (2014): apesar de não questionarem a base mais consolidada do modelo da oferta (a necessidade de um determinado nível de desenvolvimento econômico para que um país consiga conduzir um programa nuclear), estes autores argumentam que a assistência internacional não oferece contribuições significativas para que um Estado proliferador alcance a bomba. Kemp (2014) afirma que os re- 
quisitos tecnológicos já estão de tal forma difundidos, que é possível, para grande parte dos países em desenvolvimento, produzir endogenamente a bomba. Montgomery (2013) vai além, afirmando que a assistência internacional não apenas tem menos impacto do que o sugerido por autores como Kroenig (2009b) e Fuhrmann (2009a), como também pode atrapalhar o desenvolvimento nuclear do país receptor. Inspirado na pesquisa de Hymans (2012), Montgomery afirma que países com condições internas pouco favoráveis ao desenvolvimento eficiente de um programa científico de grande porte, como é o caso de um programa nuclear, não se beneficiarão de parcerias internacionais.

Fica claro, portanto, que todos os modelos apresentados oferecem contribuições importantes para a compreensão da proliferação, mas nenhum deles é isento de problemas.

\section{CONSIDERAÇÕES FINAIS}

Os modelos apresentados aqui resumem as principais tendências do debate sobre as causas da proliferação. Incorporando aspectos materiais e ideacionais, e perspectivas sistêmicas e domésticas, os modelos demonstram a complexidade do tema, inerente a qualquer fenômeno humano.

Apesar de os modelos abrangerem um largo espectro de explicações, deve ficar clara a necessidade de avanços nas pesquisas sobre as causas da proliferação, sobretudo na coleta e sistematização de evidências para os modelos e na concepção e replicação de testes de suas hipóteses. De fato, o histórico de alguns países gera ainda intensas controvérsias entre analistas. Este é o caso, por exemplo, da Índia, cuja política nuclear é atribuída, por diferentes autores, a todos os quatro modelos, conforme demonstrado. Assim, é difícil identificar quais são os fatores preponderantes na definição dos rumos da proliferação.

De qualquer forma, as pesquisas na área apontam uma conclusão fundamental: o processo de difusão da tecnologia nuclear é, inevitavelmente, complexo e multicausal. Por isso, conquanto careçamos de maior clareza sobre as condições em que cada modelo pode ser adequadamente aplicado, os modelos não necessariamente competem entre si. Eles se complementam, e fornecem as lentes necessárias para que as diferentes facetas da proliferação possam ser observadas e compreendidas.

\section{REFERÊNCIAS}

Ahmed, S. 1999. Pakistan's nuclear weapons program: turning points and nuclear choices. International Security, 23 (4): 178-204. 
Allison, G. T. 1969. Conceptual models and the Cuban Missile Crisis. The American Political Science Review, 63 (3): 689-718.

Allison, G. T.; Zelikow, P. 1971. Essence of decision: Explaining the Cuban Missile Crisis. New York: Longman.

Betts, R. K. 1977. Paranoids, pygmies, pariahs, and nonproliferation. Foreign Policy, 26: 157-183.

Braun, C., Chyba, C. F. 2004. Proliferation rings: New challenges to the Nuclear Nonproliferation Regime. International Security, 29 (2): 5-49.

Chafetz, G., Abramson, H., Grillot, S. 1996. Role theory and foreign policy: Belarussian and Ukrainian compliance with the Nuclear Nonproliferation Regime. Political Psychology, 17 (4): 727-757.

Cirincione, J. 2007. Bomb scare: The history \& future of nuclear weapons. New York: Columbia University Press.

Egeland, K. 2016. Change the incentives: Stigmatize nuclear weapons. Bulletin of the Atomic Scientists, Voices of Tomorrow, 15 March 2016. Disponível em: http:// thebulletin.org/change-incentives-stigmatize-nuclear-weapons9261? platform $=h$ ootsuite, acesso em 22 de março de 2016.

Epstein, W. 1977. Why states go - and don't go - nuclear. The Annals of the American Academy of Political and Social Science, 430: 16-28.

Flank, S. 1993. Exploding the black box: The historical sociology of nuclear proliferation. Security Studies, 3 (2): 259-294.

Frankel, B. 1993. The brooding shadow: Systemic incentives and nuclear weapons proliferation. Security Studies, 2 (3): 37-78.

Fuhrmann, M. 2008. Exporting Mass Destruction? The Determinants of Dual-Use Trade. Journal of Peace Research, 45 (5): 633-652.

Fuhrmann, M. 2009a. Spreading Temptation: Proliferation and Peaceful Nuclear Cooperation Agreements. International Security, 34 (1): 7-41.

Fuhrmann, M. 2009b. Taking a Walk on the Supply Side: The Determinants of Civilian Nuclear Cooperation. Journal of Conflict Resolution, 53 (2): 181-208.

Ganguly, S. 1999. India's pathway to Pokhran II: The prospects and sources of New Delhi's nuclear weapons program. International Security, 23 (4): 148-177. 
Ganguly, S. 2008. Nuclear stability in South Asia. International Security, 33 (2): 45-70.

Gartzke, E., Kroenig, M. 2009. A strategic approach to nuclear proliferation. Journal of Conflict Resolution, 53 (2): 151-160.

Gerring, J. 2012. Social Science methodology: A unified framework. Cambridge: Cambridge University Press.

Gilinsky, V. 2014. Nuclear Power, Nuclear Weapons - Clarifying the Links. In: Sokolski, H. (Ed.). Moving Beyond Pretense: Nuclear Power and Nonproliferation. Carlisle Barracks: United States Army War College Press, 119-148.

Goertz, G., Mahoney, J. 2012. A tale of two cultures: Qualitative and quantitative research in the Social Sciences. Princeton: Princeton University Press.

Graham Jr., T., Hansen, K. A. 2009. Preventing catastrophe: The use and misuse of intelligence in efforts to halt the proliferation of weapons of mass destruction. Stanford: Stanford University Press.

Halperin, M. H. 1972. The decision to deploy the ABM: Bureaucratic and domestic politics in the Johnson administration. World Politics, 25 (1): 62-95.

Holdren, J. P. 1983. Nuclear power and nuclear weapons: the connection is dangerous. The Bulletin of the Atomic Scientists.

Hymans, J. E. C. 2012. Achieving nuclear ambitions: Scientists, politicians and proliferation. Cambridge: Cambridge University Press.

Hymans, J. E. C. 2006a. The psychology of nuclear proliferation: Identity, emotions and foreign policy. Cambridge: Cambridge University Press.

Hymans, J. E. C. 2006b. Theories of nuclear proliferation. Nonproliferation Review, $\left.13, \mathrm{n}^{\circ} 3\right): 455-465$.

Jervis, R. 1989. The meaning of the nuclear revolution: Statecraft and the prospect of Armageddon. Ithaca: Cornell University Press.

Jesus, D. S. V. de. 2012. Em nome da autonomia e do desenvolvimento: o Brasil e a não-proliferação, o desarmamento e os usos pacíficos da energia nuclear. Meridiano 47, 13 (129): 28-34.

Jo, D.-J., Gartzke, E. 2007. Codebook and data notes for 'Determinants of nuclear weapons proliferation: a quantitative model'. Disponível em: http://pages.ucsd. edu/ egartzke/data/jo_gartzke_0207_codebk_0906.pdf, acesso em 15 nov. 2015. 
Jo, D.-J., Gartzke, E. 2007. Determinants of nuclear weapons proliferation. Journal of Conflict Resolution, 51 (1): 167-194.

Kemp, R. S. 2014. The nonproliferation emperor has no clothes: The gas centrifuge, supply-side controls, and the future of nuclear proliferation. International Security, 38 (4): 39-78.

King, G., Keohane, R., Verba, S. 1994. Designing social inquiry: Scientific inference in qualitative research. Princeton: Princeton University Press.

Klare, M. 2011. Rogue states and nuclear outlaws: America's search for a new foreign policy. New York: Hill and Wang (eBook edition).

Kroenig, M. 2009a. Exporting the bomb: Why states provide sensitive nuclear assistance. American Political Science Review, 103 (1): 113-133.

Kroenig, M. 2009b. Importing the bomb: Sensitive nuclear assistance and nuclear proliferation. Journal of Conflict Resolution, 53 (2): 161-180.

Lavoy, P. R. 1993. Nuclear myths and the causes of nuclear proliferation. Security Studies, 2 (3): 192-212.

Lee, J. H. 2016. Why Kim Jong Un needs nuclear weapons. Foreign Policy, Argument. Disponível em: http://foreignpolicy.com/2016/05/06/ why-kim-jong-un-needs-nuclear-weapons/, acesso em 10 de maio de 2016.

May, M. M. 1994. Nuclear weapons supply and demand. American Scientist, 82 (6): 526-537.

Medeiros, T. R. de. 2005. Entraves ao desenvolvimento da tecnologia nuclear no Brasil: dos primórdios da era atômica ao Acordo Nuclear Brasil-Alemanha. Dissertação (mestrado). Universidade Federal de Minas Gerais.

Monteiro, N. P., Debs, A. 2014. The strategic logic of nuclear proliferation. International Security, 39 (2): 7-51.

Montgomery, A. H. 2013. Stop helping me: When nuclear assistance impedes nuclear programs. Disponível em: http://people.reed.edu/ ahm/Projects/ProlifTK/ Montgomery2013Stop.pdf, acesso em 03 de janeiro de 2016.

Montgomery, A. H.; Sagan, S. D. 2009. The perils of predicting proliferation. Journal of Conflict Resolution, 53 (2): 302-328.

Nakatani, H. 2012. North Korea: the utilization of nuclear weapons for international prestige. The Globalized World Post. Disponível em: https://thegwpost. com/2012/07/06/848/, acesso em 09 de junho de 2016. 
Ogilvie-White, T. 1996. Is there a theory of nuclear proliferation? An analysis of the contemporary debate. The Nonproliferation Review, 4 (1): 43-60.

Oliveira, O. M. de. 1998. A integração bilateral Brasil-Argentina: tecnologia nuclear e Mercosul. Revista Brasileira de Política Internacional, 41 (1): 5-23.

O’Reilly, K. P. 2013. A Rogue Doctrine?: The role of strategic culture on US foreign policy behavior. Foreign Policy Analysis, 9 (1): 57-77.

Patti, C. 2013. O programa nuclear brasileiro entre passado e future. Boletim Meridiano 47, 14 (140): 49-55.

Pinkston, D. A. 2003. Domestic politics and stakeholders in the North Korean missile development program. The Nonproliferation Revier. Disponível em: https://www.nonproliferation.org/wp-content/uploads/npr/102pink.pdf, acesso em 09 de junho de 2016.

Price, R., Tannenwald, N. 1996. Norms and deterrence: the nuclear and chemical weapons taboo. In: Katzenstein, P. J. The culture of national security: Norms and identity in world politics. New York: Columbia University Press.

Sagan, S. D. 1996/97. Why do states build nuclear weapons: Three models in search of a bomb. International Security, 21 (3): 54-86.

Sagan, S. D., Waltz, K. N. 2003. The spread of nuclear weapons: a debate renewed. New York: W. W. Norton \& Company, Inc.

Sagan, S. D. 2004. Realist perspectives on ethical norms and weapons of mass destruction. In: Hashmi, S., Lee, S. (Ed.). Ethics and weapons of mass destruction: Religious and secular perspectives. Cambridge: Cambridge University Press, 73-95.

Sagan, S. D. 2011. The causes of nuclear weapons proliferation. Annual Review of Political Science, 14: 225-244.

Schelling, T. C. 1966. Arms and influence. New Haven: Yale University Press.

Schelling, T. C. 1980. The strategy of conflict. Cambridge: Harvard University Press.

Silva, M. V. M. da. 2010. O Tratado Sobre a Não-Proliferação de Armas Nucleares (TNP) e a inserção do Estado brasileiro no regime dele decorrente. Dissertação (mestrado). Universidade do Estado do Rio de Janeiro.

Singh, S., Way, C. R. 2004. The correlates of nuclear proliferation: A quantitative test. Journal of Conflict Resolution, 48 (6): 859- 885. 
Solingen, E. 1994. The political economy of nuclear restraint. International Security, 19 (2): 126-169.

Suchman, M. C., Eyre, D. P. 1992. Military procurement as rational myth: Notes on the social construction of weapons proliferation. Sociological Forum, 7 (1): 137-161.

Tannenwald, N. 2007. The nuclear taboo: The United States and the non-use of nuclear weapons since 1945. Cambridge: Cambridge University Press.

Thayer, B. A. 1995. The causes of nuclear proliferation and the utility of the Nuclear Nonproliferation Regime. Security Studies, 4 (3): 463-519.

Vargas, E. V. 1997. Átomos na integração: a aproximação Brasil-Argentina no campo nuclear a construção do Mercosul. Revista Brasileira de Política Internacional, 40 (1): 41-74.

Waltz, K. N. 1995. Peace, stability and nuclear weapons. IGCC-PP No. 15.

Weixing, H. 2000. New Delhi's nuclear bomb: a systemic analysis. World Affairs, 163 (1): 28-38.

Woo, J. 2015. Structural impediments, domestic politics, and nuclear diplomacy in post-Kim Il-sung North Korea. Pacific Focus, 30 (1): 59-77. 


\section{NOTAS}

1. Sobre o significado da revolução nuclear, conferir Jervis (1989). Schelling (1966) argumenta que a principal mudança decorrente do advento da bomba atômica não se refere à dimensão da destruição que pode ser engendrada, mas à sua velocidade. Nesse sentido, tecnologias anteriores a 1945 eram já perfeitamente capazes de causar milhões de mortes e ampla destruição material; mas os arsenais nucleares permitem que essas ações sejam realizadas de forma comparativamente imediata, centralizada e automatizada.

2. Um dos principais debates da área diz respeito não às causas da proliferação, mas às suas consequências. Este debate opôs otimistas a pessimistas, os primeiros percebendo na difusão de armamentos nucleares um elemento capaz de promover maior estabilidade no sistema internacional, e os segundos vendo como consequência da proliferação o aumento do risco de conflitos nucleares no mundo. Esse debate foi consolidado no famoso livro de Scott Sagan e Kenneth Waltz (2003).

3. Os países que hoje detêm capacidade nuclear são Estados Unidos, Rússia, Reino Unido, França, China, Israel, Índia, Paquistão e Coreia do Norte. Dentre os países que exploraram a opção nuclear estão a Líbia, o Irã e a Coreia do Sul. A África do Sul chegou a produzir a bomba, mas desmantelou seu arsenal no começo da década de 1990.

4. Marcos Valle Machado da Silva (2010) aborda o tema das causas da proliferação, mas sua análise se fundamenta apenas no modelo desenvolvido por Kenneth Waltz (1995), e não explora outras interpretações sobre o tema.

5. Optamos por empregar o termo "modelo" aqui de forma semelhante à adotada por Sagan (1996/97), para referir-nos a grupos de abordagens sobre a proliferação que compartilham alguns elementos centrais. Assim, um mesmo modelo pode abranger contribuições de autores que não apresentam plena concordância, mas que convergem sobre algum aspecto fundamental acerca das forças motrizes da proliferação. Portanto, o termo não é empregado no sentido estrito de modelos formais de análise científica.

6. A inclusão que Cirincione (2007) faz da economia como um fator explicativo da proliferação nuclear é questionável. Considerações econômicas funcionam, fundamentalmente, como um obstáculo à decisão de um Estado para iniciar um programa nuclear, devido a seus custos muito elevados. Nas palavras do próprio autor: "Nuclear weapons are big ticket items. They and their delivery systems are expensive to make. Economic considerations alone cannot explain a state's pursuit 
of nuclear weapons. A country does not launch a nuclear program just because it can afford one. Nor will economic costs have much impact if a state decides nuclear weapons are vital to its national security." (Cirincione 2007, 76-77). Portanto, optamos aqui por não incorporar o fator econômico como um modelo específico em nosso panorama das causas da proliferação. É importante notar, no entanto, que a capacidade que atores domésticos politicamente bem posicionados venham a ter de convencer os tomadores de decisão de que os custos serão pequenos comparados aos benefícios (e, sobretudo, de subestimar os custos reais do programa) pode ser fundamental para que o programa nuclear seja iniciado. Considerações econômicas são, então, contempladas pelo modelo acerca do impacto da política doméstica sobre a proliferação.

7. Utilizamos o termo causa aqui de forma permissiva. Evidentemente, é muito difícil tratar de relações causais em fenômenos políticos e em interações sociais; nos referimos, então, às condições que propiciam a proliferação, bem como às motivações dos Estados para proliferarem, ou seja, àqueles elementos que permitem e facilitam a proliferação. Sobre a causalidade nas Ciências Sociais, conferir King, Keohane e Verba (1994, cap3), Gerring (2012, part III) e Goertz e Mahoney (2012, part I).

8. Note-se que tanto as normas e ideias compartilhadas quanto a política doméstica constituem elementos que impactam sobre a demanda dos Estados pela bomba. Assim, podemos considerar que temos três modelos que olham para a demanda e um modelo que olha para a oferta.

9. Esta divisão é muito similar à apresentada por Thayer (1995).

10. De fato, o longo histórico de rivalidade entre a Índia e o Paquistão resultou em um grande peso da capacidade nuclear indiana para as decisões políticas paquistanesas na área nuclear. Sobre essa relação, conferir Ahmed (1999) e Ganguly (2008).

11. No original: "Nations that have made a positive nuclear-weapons decision have either perceived a threat to the survival of their state that no other state could credibly deter, or faced a security situation where, with a nuclear-weapons capability, they could significantly increase their voice in matters that mattered to survival."

12. De fato, segundo Monteiro e Debs (2014, 21), quase todos os países que exploraram seriamente a possibilidade de adquirir a bomba enfrentavam ameaças sérias a sua segurança, sendo o Brasil, a Argentina e a Romênia as únicas exceções.

13. No original: “[ $\ldots]$ a jointly recognized expectation that [nuclear weapons $]$ may not be used [...]".

14. Este é o segundo modelo de análise de Allison e Zelikow (1971), referente aos processos organizacionais. 
15. Este é o terceiro modelo de análise de Allison e Zelikow (1971), referente à política governamental.

16. No original: "Men share power. Men differ concerning what must be done. The differences matter. This milieu necessitates that policy be resolved by politics. What the nation does is sometimes the result of the triumph of one group over others."

17. A política doméstica norte-coreana parece ser, de fato, fundamental para se compreender as políticas de defesa adotadas pelo país. Para uma análise sobre como as interações domésticas afetam também o programa de desenvolvimento de mísseis da Coreia do Norte, conferir Pinkston (2003).

18. Cooperação é aqui entendida como o estabelecimento de parcerias que viabilizam a transferência de tecnologia e de know-how. Não se trata da cooperação realizada no sentido de estabelecer acordos e regimes de não proliferação.

19. No original: "Because states stand to gain by possessing nuclear weapons, the supply-side factors that enable nuclear development are among the most important determinants of nuclear proliferation."

20. Particularmente, instalações para enriquecimento de urânio ou reprocessamento de plutônio.

21. No original: " $[\ldots]$ since all nuclear commodities are dual-use in nature, importing states can use the technology and knowledge they acquire to build nuclear weapons."

22. As conclusões a que Kroenig (2009b) e Fuhrmann (2009a) chegam propiciam o questionamento sobre as razões que levariam potências nucleares a firmarem acordos de cooperação nuclear com outros Estados, uma vez que isso poderia contribuir para a proliferação. Kroenig (2009a) e Fuhrmann (2009b) desenvolvem explicações semelhantes sobre esse ponto. Ambos os autores argumentam que considerações de segurança podem determinar as parcerias nucleares, de tal forma que, sob certas circunstâncias, a proliferação nuclear não seria inteiramente imprevista ou indesejada. Assim, a cooperação seria mais provável, por exemplo, entre Estados que têm inimigos em comum. 


\section{POR QUE OS ESTADOS QUEREM A BOMBA?}

AS CONDIÇÕES PARA A PROLIFERAÇÃO NUCLEAR

\section{RESUMO}

Este artigo tem por objetivo apresentar uma revisão da literatura sobre os condicionantes da proliferação nuclear. Reunimos as diferentes abordagens em quatro grandes modelos: a demanda dos Estados por segurança, o contexto normativo e ideacional, os determinantes políticos domésticos e a oferta de tecnologia.

Palavras-chave: Proliferação; Armamentos nucleares; Tecnologia nuclear; Demanda e oferta.

\section{ABSTRACT}

This article aims to present a review of the literature on the determinants of nuclear proliferation. We bring together the different approaches into four umbrella models: the States' demand for safety, normative and ideational contexts, domestic political determinants and the provision of technology.

Keywords: Proliferation; Nuclear Weapons; Nuclear Technology; Demand and Supply. 